ARTigo Original

Original ARTicle

\title{
Avaliação dos Óbitos e Necropsias em Pacientes Internados em um Serviço de Reumatologia Pediátrica por um Período de Dez Anos ${ }^{(*)}$
}

\section{Deaths and Necropsies Evaluation in Hospitalized Patients of a Pediatric Rheumatolosy Unit for a Period of Ten Years}

\author{
Mércia Moreira Facó(1), Lucia Akemi Nukumizu ${ }^{(2)}$, Ana Júlia Pantoja de Moraes ${ }^{(1)}$, Paula Cruz Borreli Barros ${ }^{(2)}$, \\ Eduardo Juan Troster ${ }^{(3)}$, Clovis Artur Almeida da Silva ${ }^{(4)}$
}

\section{RESUMO}

Objetivo: correlacionar os dados clínicos do óbito com achados de necropsia em pacientes internados em uma unidade de reumatologia pediátrica. Métodos: o estudo é uma coorte histórica. Em dez anos, no período compreendido entre janeiro de 1994 e dezembro de 2003, ocorreram 57.159 internações com 1.907 (3\%) óbitos no Instituto da Criança. Destas internações, 548 (1\%) apresentaram doenças reumáticas ou pediátricas e foram acompanhadas pela Unidade de Reumatologia Pediátrica, incluindo 348 pacientes. Os óbitos e as necropsias foram analisados e a classificação de Goldman foi utilizada para detectar discordância entre o diagnóstico clínico do óbito e a necropsia. Resultados: no período do estudo, 34 (10\%) pacientes evoluíram para óbito. As principais doenças associadas ao óbito foram: lúpus eritematoso sistêmico juvenil (LESJ) em 18 pacientes (53\%) e artrite idiopática juvenil (AIJ) em sete casos (21\%). Necropsias foram realizadas em 21 pacientes (64\% dos óbitos). Nos 18 casos de LESJ, a atividade da doença esteve presente em 16 casos, sendo associada à septicemia em 15 deles. Discordância entre o diagnóstico clínico e necropsia foi evidenciada em seis casos com LESJ: três com infecções fúngicas, um com tuberculose, um com nefrite proliferativa difusa e outro com aterosclerose. Sete pacientes com AIJ evoluíram para óbito: septicemia em quatro e síndrome de ativação macrofágica em três. Em um caso de AIJ foi diagnosticado, exclusivamente na necropsia: linfoma de Hodgkin, aterosclerose e infarto anterior do miocárdio. Conclusões: a freqüência de óbitos foi $10 \%$ e necropsia evidenciou doença infecciosa, aterosclerose ou neoplasia não diagnosticadas previamente. A necropsia é importante

\section{ABSTRACT}

Objective: to correlate the clinical data of necropsies in hospitalized patients of a Pediatric Rheumatology Unit. Methods: this study is a historic cohort. In ten years, from January 1994 to December 2003, there were 57,159 hospitalizations with 1,907 (3\%) deaths in Instituto da Criança. From these hospitalizations, $548(1 \%)$ presented rheumatic or pediatric diseases and were followed by the Pediatric Rheumatology Unit, involving 348 patients. The deaths and necropsies were analyzed and the Goldman Classification was used to detect any disagreement between the clinic diagnosis and the necropsy. Results: over the period of this study, 34 (10\%) of patients died. The main diseases that caused deaths were: juvenile systemic erithematosus lupus (JSLE) in 18 patients (53\%) and juvenile idiopathic arthritis (JIA) in 7 cases (21\%). Necropsies were done in 21 patients (64\% of deaths). In 18 cases of JSLE, disease activity was present in 16 cases, being associated to septicemia in 15. Differences between the clinical diagnosis and necropsy were observed in 6 cases of JSLE: 3 with fungal infection, 1 with tuberculosis, 1 with diffuse proliferative nephritis and 1 with atherosclerosis. Seven patients with JIA died: septicemia in 4 and macrophage activation syndrome in 3. In 1 case of JIA were detected only by necropsy: Hodgkin lymphoma, atherosclerosis and anterior myocardial infarction. Conclusions: the frequency of deaths was 10\% and necropsy showed infection disease, atherosclerosis or malignancy not detected previously. Necropsy is important to determine events

\footnotetext{
* Trabalho realizado nas Unidades de Reumatologia Pediátrica e Terapia Intensiva do Instituto da Criança do Hospital das Clínicas da Faculdade de Medicina da Universidade de São Paulo (ICr-HC-FMUSP), São Paulo-SP, Brasil. Recebido em 02/09/2004. Aprovado, após revisão, em 20/01/2005.

1. Médica pós-graduanda do Departamento de Pediatria da FMUSP.

2. Médica colaboradora da Unidade da Reumatologia Pediátrica do Departamento de Pediatria da FMUSP.

3. Doutor em Medicina pela FMUSP. Chefe da Unidade de Terapia Intensiva do Departamento de Pediatria da FMUSP.

4. Doutor em Medicina pela FMUSP. Chefe da Unidade de Reumatologia Pediátrica do Departamento de Pediatria da FMUSP.

Endereço para correspondência: Dr. Clóvis Artur Almeida da Silva. R. Senador Cesar Lacerda Vergueiro, 494/82, V. Madalena, CEP 05435-010, São Paulo, SP, Brasil. Tel. (1 1) 3069-8675; fax (11) 3069-8510; e-mail: clovisaas@icr.hcnet.usp.br
} 
para determinar eventos não esclarecidos ou duvidosos no óbito e deve ser sempre solicitada.

Palavras-chave: reumatologia pediátrica, lúpus eritematoso sistêmico juvenil, artrite idiopática juvenil, mortalidade, necropsia,

\section{INTRODUÇÃO}

Os serviços de reumatologia pediátrica acompanham crianças e adolescentes com várias doenças e prognósticos diversos, particularmente: artrite idiopática juvenil (AIJ), lúpus eritematoso sistêmico juvenil (LESJ), dermatomiosite juvenil (DMJ), febre reumática e vasculites.

$\mathrm{Na}$ última década, o maior reconhecimento das doenças reumatológicas pediátricas vem promovendo diagnósticos e encaminhamentos mais precoces. Por sua vez, os avanços terapêuticos também permitiram a melhora na sobrevida desses pacientes. Por serem geralmente doenças graves, com períodos de recorrência e remissão, necessitam freqüentemente de internações hospitalares, principalmente para controle da atividade da doença e ou tratamento de infecções associadas.

Óbitos ocorrem, habitualmente, por atividade e/ou infecção, principalmente, no LESJ, AIJ e DMJ. No LESJ, a mortalidade costuma ocorrer em dois períodos: no início da doença, relacionada à atividade e infecção, e tardiamente, por infecção e danos cumulativos decorrentes da própria doença ou dos medicamentos utilizados para o seu tratamento ${ }^{(1,2)}$.

Os estudos de necropsias são importantes para determinar eventos não esclarecidos ou duvidosos do óbito. $\mathrm{Na}$ faixa etária pediátrica, estes são raramente descritos e comumente realizados como relato de caso $^{(3)}$. Nos adultos com LES, a realização de necropsias evidenciou achados no exame anátomo-patológico não suspeitados na prática clínica, particularmente associados com dano cumulativo da doença ou evento adverso das drogas utilizadas, como exemplo, a aterosclerose com envolvimento de coronárias, aorta e vasos cerebrais. A aterosclerose tem sido apontada como uma importante causa de morbi-mortalidade principalmente após o advento do uso de corticoesteróides ${ }^{(2,4)}$.

O objetivo deste trabalho foi avaliar os óbitos e necropsias em pacientes internados com doenças reumáticas nas diversas enfermarias do ICr do Hospital das Clínicas (HC) da FMUSP e seguidos pela Unidade de Reumatologia Pediátrica, assim como estudar a discordância entre o diagnóstico clínico do óbito e o anátomo-patológico da necropsia. not detected or doubtful in death patients and must always be requested.

Keywords: pediatric rheumatology, juvenile systemic lupus erithematosus, juvenile idiopathic arthritis, mortality, necropsy.

\section{PACIENTES E MÉTODOS}

O estudo é uma coorte histórica. Em dez anos, no período compreendido entre janeiro de 1994 e dezembro de 2003, ocorreram 57.159 internações no ICr do HC-FMUSP. Nesse período, 548 (1\%) internações foram acompanhadas pela Unidade de Reumatologia Pediátrica, incluindo 348 crianças e adolescentes internados nas seguintes enfermarias: especialidade, pronto socorro, unidade semi-intensiva, unidade de terapia intensiva (UTI), cirurgia pediátrica ou berçário de alto risco. Nestes pacientes foram avaliados os óbitos e as necropsias. Foram incluídos apenas os pacientes que evoluíram para óbito durante internação nesse Hospital Universitário.

As internações para realização de procedimentos como: biópsia (renal, cutânea, hepática, pulmonar e ganglionar) e utilização de medicamentos endovenosos na enfermaria de leito dia (pulsoterapia com metilprednisolona, ciclofosfamida e gamaglobulina) foram excluídas.

Os pacientes preencheram critérios de classificação do Colégio Americano de Reumatologia para LESJ ${ }^{(5)}$ e para a síndrome de Churg-Strauss ${ }^{(6)}$. O critério utilizado para DMJ foi de Bohan and Peter, $1975^{(7)}$ e para AIJ foi o critério do International League of Associations for Rheumatology $(\text { ILAR })^{(8)}$.

A biópsia renal dos pacientes com LESJ foi avaliada em conformidade com as classes histológicas de I a VI da Organização Mundial de Saúde (OMS) ${ }^{(9)}$. Na última internação também foram analisados: atividade do LESJ avaliada pelo Systemic Lupus Erithematosus Disease Activity Index (SLEDAI) ${ }^{(10)}$, sendo considerada arbitrariamente como atividade da doença valores numéricos acima ou igual a $8^{(10)}$; curso da doença (dividido em: remissivo, remissivo-recidivante e crônico ativo ${ }^{(11)}$ ) e dano cumulativo causado pelo LESJ e/ou pelo seu tratamento (Systemic Lupus International Collaborating Clinics/ACR [SLICC/ACR] Damage Index [SLICC-ACR-DI]) $)^{(12)}$.

$\mathrm{O}$ curso da AIJ foi dividido em remissivo (doença inativa com duração mínima de dois anos) e policíclico (doença com períodos de atividade e inatividade $)^{(13)}$. 
Neste estudo, infecção hospitalar foi considerada como qualquer infecção adquirida após 48 a 72 horas do internamento, de acordo com os critérios da Comissão de Controle de Infecção Hospitalar do Instituto da Criança ${ }^{(14)}$.

As necropsias foram realizadas pela Disciplina de Patologia da FMUSP e previamente autorizadas pelos pais ou responsáveis. Estas foram classificadas em três tipos: A, B e C. A necropsia do tipo A foi realizada por médicos residentes e assistentes empregando a técnica de evisceração total de Rokitansk ${ }^{(15)}$ com avaliação completa da microscopia de todas as vísceras. A necropsia do tipo B foi também realizada pelos médicos residentes e assistentes, porém, com retirada e avaliação microscópica das principais vísceras relacionadas à doença de base. A necropsia do tipo $\mathrm{C}$ foi realizada apenas pelo médico assistente, com retirada das principais vísceras; a microscopia era realizada se houvesse lesão macroscópica.

A classificação de Goldman ${ }^{(16)}$ foi utilizada para detectar discordância entre o diagnóstico clínico do óbito e o anátomo-patológico da necropsia (Tabela 1). Nesta classificação, o autor divide os diagnósticos em "principais" (relacionados à doença de base ou causa imediata do óbito) e "secundários" (incluindo condições prévias ou causas contribuintes para o óbito) e também avalia o impacto no prognóstico e tratamento (se o conhecimento prévio dos dados anátomopatológicos modificaria a conduta terapêutica e prognóstico).

Neste estudo, o diagnóstico clínico do óbito foi determinado através dos registros do prontuário, atestado de óbito ou da solicitação da necropsia. Nos pacientes que apresentaram duas ou mais classes de Goldman, a classe considerada foi aquela de maior impacto na terapêutica e/ou no prognóstico.

O projeto foi aceito pelo Comitê de Pesquisa e Ética do HC-FMUSP (CAPPesq número 520/03).

TABELA 1

ClassificaÇão de GoldMAn PARA A CONCORDÂNCIA ENTRE O DIAGNÓSTICO CLÍNICO DO ÓBITO E ANÁTOMO-PATOLÓGICO DA NECROPSIA

\begin{tabular}{lcc}
\hline $\begin{array}{l}\text { Classificação de } \\
\text { Goldman }\end{array}$ & $\begin{array}{c}\text { Diagnóstico clínico } \\
\text { discordante da necropsia }\end{array}$ & $\begin{array}{c}\text { Impacto no } \\
\text { prognóstico }\end{array}$ \\
\hline Classe I & Principal & Positivo \\
\hline Classe II & Principal & Negativo \\
Classe III & Secundário & Negativo \\
Classe IV & Secundário & Positivo \\
Classe V & $*$ & $*$ \\
\hline
\end{tabular}

${ }^{*}$ Os diagnósticos clínicos e os achados da necropsia foram concordantes; Positivo $=0$ conhecimento dos dados da necropsia modificaria a terapêutica e o prognóstico; Negativo $=0$ conhecimento dos dados da necropsia não modificaria a terapêutica e o prognóstico

\section{RESULTADOS}

No período do estudo, 1.907 óbitos ocorreram nas diversas enfermarias do ICr do HC-FMUSP. Desses, 34 óbitos ocorreram em doenças reumáticas, o que corresponde a $2 \%$ do total de óbitos e 9,5\% dos 348 pacientes seguidos nesse serviço.

As principais doenças associadas ao óbito foram: LESJ em 18 casos (53\%), AIJ em 7 (21\%) e doença de Weber Christian em 2 (6\%). Um óbito ocorreu em cada uma das seguintes doenças: DMJ, síndrome de Churg-Strauss, síndrome do anticorpo antifosfolípide associada a doenças auto-imunes (tireoidite e glomerulonefrite), fibrose retroperitonial, vasculite necrosante por meningoccemia, eritema nodoso associado a histiocitose das células de Langerhans e amiloidose por síndrome articular infantil, crônica, cutânea e neurológica (síndrome CINCA). Necropsias foram realizadas em 21 pacientes (64\% dos óbitos). As Tabelas 2, 3 e 4 mostram os dados referentes aos óbitos de acordo com as principais doenças. O principal local de internação destes casos foi a UTI em 28 pacientes (85\%).

A Tabela 2 inclui as características dos óbitos e necropsias em 18 pacientes com LESJ. Destes casos, a idade na época do óbito variou de 4,1 a 21,2 anos (média de 13,8 anos) e a duração da doença variou de um mês a 8,7 anos (média de 2,8 anos). Atividade da doença (SLEDAI $\geq 8$ ) foi a causa clínica do óbito em 16 casos (83\%) sendo associada à septicemia em 15 destes. Quatro pacientes evoluíram para óbito no primeiro mês de doença por atividade associada à infecção. Onze pacientes apresentavam nefrite lúpica, sendo que 10 evoluíram para insuficiência renal aguda, destes, 4 pertenciam à classe IV da OMS. O diagnóstico da nefrite foi estabelecido apenas na necropsia em 2 pacientes (casos 12 e 13). As principais terapias utilizadas para a atividade do LESJ durante a última internação de cada paciente foram: pulsoterapia com metilprednisolona $(30 \mathrm{mg} / \mathrm{kg} / \mathrm{dia}$, por três dias) em 16 casos, pulsoterapia com ciclofosfamida (500 a $1000 \mathrm{mg} / \mathrm{m}^{2}$ ) em oito, plasmaférese em cinco e imunoglobulina endovenosa $(2,0 \mathrm{~g} / \mathrm{kg}$ em dose única administrada em 10 horas) em quatro.

Infecções no LESJ foram diagnosticadas em 16 pacientes, sendo associada à atividade em 15 deles. Agentes infecciosos foram isolados em culturas de sangue, urina, catéter, líquor ou infecção cutânea em 12 casos: Staphilococcus aureus em nove, Pseudomonas aeruginosa em três e Candida albicans em três. Antibioticoterapia foi utilizada em 16 pacientes com septicemia, preferencialmente ceftriaxone e vancomicina. Terapia anti-fúngica foi utilizada em cinco casos: fluconazol em três, anfotericina B em um e uma 
paciente recebeu associação destas drogas. Esta terapia foi realizada empiricamente nas pacientes 7 e 10 que, respectivamente, apresentaram na necropsia miocardite fúngica e aspergilose pulmonar. Uma paciente (caso 16), que não realizou necropsia, apresentou infecção por Criptococcus neoformans.

TABELA 2

Características dos óbitos e necropsias em 18 pacientes com lúpus eritematoso sistêmico juvenil

\begin{tabular}{|c|c|c|c|c|c|c|c|c|}
\hline $\mathrm{N}$ & IA & TD & $S$ & $\begin{array}{l}\text { SLEDAI /Curso da doença/ } \\
\text { SLICC ACR DI }\end{array}$ & $\mathrm{TI}$ & Causa clínica do óbito & $\begin{array}{l}\text { Achados da } \\
\text { necropsia }\end{array}$ & $\begin{array}{l}\text { Classe de } \\
\text { Goldman }\end{array}$ \\
\hline 1 & $15,6 \mathrm{a}$ & $5,6 a$ & $\mathrm{~F}$ & $18 / \mathrm{CA} / 0$ & $14 d$ & $\begin{array}{c}\text { Infecção (septicemia IH) } \\
\text { Atividade (vasculite SNC, nefrite II) }\end{array}$ & Não realizada & NA \\
\hline 2 & $3,9 a$ & $1 \mathrm{~m}$ & $\mathrm{~F}$ & 23/NA/NA & $36 d$ & $\begin{array}{l}\text { Infecção (septicemia IH) } \\
\text { Atividade (IRA) }\end{array}$ & $\begin{array}{l}\text { Septicemia } \\
\text { HP }\end{array}$ & V \\
\hline 3 & $12,6 a$ & $3,6 a$ & $\mathrm{~F}$ & $11 / \mathrm{CA} / 0$ & $3 d$ & $\begin{array}{c}\text { Infecção (septicemia) } \\
\text { Atividade (IRA, pleurite) }\end{array}$ & Não realizada & NA \\
\hline 4 & $10,8 a$ & $1 \mathrm{~m}$ & $\mathrm{~F}$ & $\begin{array}{l}\text { 14/NR NA/ } \\
\text { NR NA }\end{array}$ & $12 d$ & $\begin{array}{c}\text { Infecção (septicemia IH) } \\
\text { Atividade }\end{array}$ & $\begin{array}{l}\text { Septicemia } \\
\text { Hemopericárdio }\end{array}$ & V \\
\hline 5 & $14,3 a$ & $1,8 a$ & $\mathrm{~F}$ & $22 / \mathrm{CA} / 1$ & $35 d$ & $\begin{array}{l}\text { Infecção (septicemia IH) } \\
\text { Atividade (nefrite IV II, } \\
\text { IRA, HP, pericardite) }\end{array}$ & $\begin{array}{l}\text { Septicemia } \\
\text { HP } \\
\text { Pericardite }\end{array}$ & V \\
\hline 6 & $15,3 a$ & $4 \mathrm{~m}$ & $\mathrm{~F}$ & 12/NA/NA & $30 d$ & $\begin{array}{c}\text { Infecção (septicemia) } \\
\text { Atividade (vasculite SNC, nefrite II) }\end{array}$ & Não realizada & NA \\
\hline 7 & $12,6 a$ & $1 \mathrm{~m}$ & $\mathrm{~F}$ & $18 / \mathrm{CA} / 1$ & $50 d$ & $\begin{array}{c}\text { Infecção (septicemia IH) } \\
\text { Atividade (nefrite IV, IRA, } \\
\text { vasculite SNC, pleurite, pericardite) }\end{array}$ & $\begin{array}{l}\text { Miocardite fúngica } \\
\text { EI Pleurite/ } \\
\text { Pericardite }\end{array}$ & II \\
\hline 8 & $16,6 a$ & $4,6 a$ & M & $10 / \mathrm{CA} / 0$ & $3 d$ & $\begin{array}{c}\text { Infecção (septicemia) } \\
\text { Atividade (nefrite IV, IRA) }\end{array}$ & Não realizado & NA \\
\hline 9 & $18,4 a$ & $4,5 a$ & $\mathrm{~F}$ & $28 / \mathrm{CA} / 0$ & $60 d$ & $\begin{array}{c}\text { Infecção (septicemia IH) } \\
\text { Atividade (nefrite IV, IRA, } \\
\text { pericardite) }\end{array}$ & $\begin{array}{l}\text { Septicemia } \\
\text { Pericardite } \\
\text { PAA }\end{array}$ & III \\
\hline 10 & 10,1 & $1 \mathrm{~m}$ & $\mathrm{~F}$ & $11 / \mathrm{NA} / \mathrm{NA}$ & $60 d$ & $\begin{array}{c}\text { Infecção (septicemia IH) } \\
\text { Atividade (nefrite, IRA, } \\
\text { pericardite) }\end{array}$ & $\begin{array}{l}\text { Septicemia } \\
\text { AP } \\
\text { Nefrite III }\end{array}$ & II \\
\hline 11 & $4,1 \mathrm{a}$ & $11 \mathrm{~m}$ & M & $0 / \mathrm{CA} / 0$ & $1 d$ & Infecção (septicemia) & Não realizada & NA \\
\hline 12 & $21,2 \mathrm{a}$ & $2,8 a$ & $\mathrm{~F}$ & $10 / \mathrm{CA} / 1$ & $14 d$ & $\begin{array}{l}\text { Infecção (septicemia IH) } \\
\text { Atividade (SNC, coma) }\end{array}$ & $\begin{array}{l}\text { Septicemia } \\
\text { TBM } \\
\text { Nefrite } \\
\text { PAA }\end{array}$ & I \\
\hline 13 & $13,6 a$ & $7 m$ & $\mathrm{~F}$ & $10 / \mathrm{CA} / 0$ & $2 d$ & $\begin{array}{c}\text { Infecção (septicemia) } \\
\text { Atividade (HP e hematúria) } \\
\text { IRA }\end{array}$ & $\begin{array}{l}\text { Septicemia } \\
\text { HP } \\
\text { Nefrite IV }\end{array}$ & III \\
\hline 14 & $16,4 a$ & $5 a$ & $\mathrm{~F}$ & $9 / \mathrm{CA} / 3$ & $5 d$ & $\begin{array}{c}\text { Infecção (septicemia) } \\
\text { Atividade (SAF, nefrite, IRA) }\end{array}$ & $\begin{array}{l}\text { Septicemia } \\
\text { EC } \\
\text { Nefrite }\end{array}$ & V \\
\hline 15 & $18,9 a$ & $3,7^{\mathrm{a}}$ & $\mathrm{F}$ & $18 / \mathrm{CA} / 2$ & $23 d$ & $\begin{array}{l}\text { Infecção (septicemia IH) } \\
\text { Atividade (pericardite) }\end{array}$ & $\begin{array}{l}\text { Septicemia } \\
\text { AD }\end{array}$ & I \\
\hline 16 & $16,6 a$ & $5 a$ & $\mathrm{~F}$ & $20 / \mathrm{CA} / 0$ & $92 \mathrm{~d}$ & $\begin{array}{l}\text { Infecção (NCC, septicemia IH ) } \\
\text { Atividade (nefrite IV, IRA) }\end{array}$ & Não realizada & NA \\
\hline 17 & $15 a$ & $3 a$ & $\mathrm{~F}$ & $16 / \mathrm{CA} / 1$ & $12 d$ & Atividade (SNC, convulsões) & Não realizada & NA \\
\hline 18 & $15,9 a$ & $8,7 a$ & $\mathrm{~F}$ & $9 / \mathrm{CA} / 2$ & $8 d$ & Atividade: plaquetopenia & Não realizada & NA \\
\hline
\end{tabular}

$\mathrm{a}=$ anos; $\mathrm{AD}=$ aspergilose difusa; $\mathrm{AP}=$ aspergilose pulmonar; $\mathrm{CA}=$ crônico ativo; $\mathrm{d}=$ dias; $\mathrm{EC}=$ edema cerebral; $\mathrm{EI}=$ endocardite infecciosa; $\mathrm{F}=$ feminino; $\mathrm{HP}=$ hemorragia pulmonar; $\mathrm{IA}=$ idade atual; $\mathrm{IH}=$ intra-hospitalar; $\mathrm{IRA}=$ insuficiência renal aguda; $\mathrm{m}=$ meses; $\mathrm{N}=$ número do paciente; $\mathrm{NA}=$ não aplicável; $\mathrm{NCC}=$ neurocriptococose; nefrite II = glomerulonefrite mesangial; nefrite III = glomerulonefrite proliferativa focal; nefrite IV = glomerulonefrite proliferativa difusa; PAA = placa de ateroma de aorta; $\mathrm{S}=$ sexo; $\mathrm{SAF}=$ síndrome do anticorpo antifosfolípide; $\mathrm{SNC}=$ sistema nervoso central; TBM = tuberculose miliar; TD = tempo de doença; TI = tempo de internação 
TABELA 3

Características dos óbitos e necropsias em sete pacientes com artrite idiopática juvenil

\begin{tabular}{|c|c|c|c|c|c|c|c|c|}
\hline $\mathrm{N}$ & IA & $S$ & TI & TD & Tipo de início/ & Causa clínica do & Achados da necropsia & Classe de Goldman \\
\hline 1 & $15 a$ & M & $6 \mathrm{~d}$ & $11,3 a$ & $\begin{array}{c}\text { Sistêmico/poliarticular } \\
\text { FR negativo }\end{array}$ & SAM & PMP & $\mathrm{V}$ \\
\hline 2 & $12,6 a$ & M & $8 d$ & $9,6 a$ & Sistêmico/sistêmico & SAM & $\begin{array}{c}\text { Esplenite } \\
\text { Esteatose hepática } \\
\text { CP }\end{array}$ & V \\
\hline 3 & $18,2 \mathrm{a}$ & M & $12 d$ & $10,9 a$ & Sistêmico/sistêmico & SAM & $\begin{array}{c}\text { PC } \\
\text { DP } \\
\text { IRA }\end{array}$ & V \\
\hline 4 & $19 a$ & M & $15 d$ & $9,2 a$ & Sistêmico/sistêmico & Septicemia IH & $\begin{array}{c}\text { Septicemia } \\
\text { PAA }\end{array}$ & III \\
\hline 5 & $4 a$ & M & $18 \mathrm{~d}$ & $2 a$ & Sistêmico/sistêmico & $\begin{array}{l}\text { Septicemia IH } \\
\text { IHA }\end{array}$ & $\begin{array}{c}\text { Septicemia } \\
\text { HCD (sais de ouro) }\end{array}$ & V \\
\hline 6 & $12,6 a$ & $\mathrm{~F}$ & $30 d$ & $5,5 a$ & Sistêmico/sistêmico & $\begin{array}{l}\text { Septicemia IH } \\
\text { Pericardite }\end{array}$ & Pericardite constrictiva & NA \\
\hline 7 & $16,7 a$ & $\mathrm{~F}$ & $8 d$ & $12,7 \mathrm{a}$ & $\begin{array}{l}\text { Sistêmico/poliarticular } \\
\text { FR negativo }\end{array}$ & $\begin{array}{l}\text { ICC } \\
\text { HP }\end{array}$ & $\begin{array}{c}\text { ICC } \\
\text { HP } \\
\text { IAM prévio } \\
\text { LNHB } \\
\text { PAG }\end{array}$ & III \\
\hline
\end{tabular}

$\mathrm{a}=$ anos; $\mathrm{CP}=$ congestão pulmonar; $\mathrm{d}=$ dias; $\mathrm{DP}=$ derrame pleural; $\mathrm{F}=$ feminino; $F \mathrm{R}=$ fator reumatóide; $\mathrm{IA}=$ idade atual; $\mathrm{HCD}=$ hepatite crônica por drogas; $\mathrm{HP}=$ hipertensão pulmonar; IAM = infarto agudo do miocárdio; ICC = insuficiência cardíaca congestiva; IHA = insuficiência hepática; IH = intra-hospitalar; IRA = insuficiência renal; $L$ LHB = linfoma não Hodking de células $\mathrm{B} ; \mathrm{m}=$ meses; $\mathrm{M}=$ masculino; $\mathrm{N}=$ número do paciente; $\mathrm{NA}=$ não aplicável; $\mathrm{PAA}=$ placa de ateroma de aorta; $\mathrm{PAG}=$ placa de ateroma generalizada; $\mathrm{PC}=$ pericardite crônica; $\mathrm{PMP}=$ ploriferação macrofágica pulmonar; $\mathrm{SAM}=$ síndrome de ativação macrofágica; $\mathrm{S}=\mathrm{sexo} ; \mathrm{TD}=$ tempo de doença; $\mathrm{TI}=$ tempo de internação

TABELA 4

Características dos óbitos e necropsias em dez pacientes com outras doenças reumatológicas pediátricas

\begin{tabular}{|c|c|c|c|c|c|c|c|c|}
\hline $\mathrm{N}$ & Doença & IA & $S$ & $\mathrm{TI}$ & TD & Causa clínica do óbito & Achados da necropsia & Classe de Goldman \\
\hline 1 & Doença de Weber Christian & $4,3 a$ & $\mathrm{~F}$ & $12 d$ & $7 a$ & $\begin{array}{l}\text { Septicemia IH } \\
\text { Atividade }\end{array}$ & Não realizada & NA \\
\hline 2 & $\mathrm{HCL}$ com eritema nodoso & $1,8 a$ & $\mathrm{~F}$ & $30 d$ & $6 a$ & Disseminação tumoral & Não realizada & NA \\
\hline 3 & Doença de Weber Christian & $11 a$ & $\mathrm{~F}$ & $20 d$ & $6 a$ & Septicemia IH & Septicemia & V \\
\hline 4 & DMJ & $7 a$ & $\mathrm{~F}$ & $12 d$ & $2,8 a$ & $\begin{array}{c}\text { Septicemia IH } \\
\text { Vasculite cutânea }\end{array}$ & $\begin{array}{c}\text { Septicemia } \\
\text { Vasculite cutânea }\end{array}$ & V \\
\hline 5 & Sindrome CINCA & $15 a$ & M & $8 d$ & $7,1 \mathrm{a}$ & Septicemia IH & SepticemiaAmiloidosePAA & I \\
\hline 6 & Fibrose RP & $19,2 \mathrm{a}$ & M & $11 d$ & $9 a$ & Septicemia IH & Não realizada & NA \\
\hline 7 & SAF & $7,5 a$ & M & $50 d$ & $6 a$ & Septicemia IHHPTC AVCI & SepticemiaHPTireoidite & V \\
\hline 8 & Sindrome de Churg-Strauss & $7,7 a$ & M & $8 d$ & $8 m$ & Vasculite SNC Coma & Não realizada & NA \\
\hline 9 & $\begin{array}{l}\text { Vasculite necrosante por } \\
\text { infecção meningocócica }\end{array}$ & $2,4 a$ & $\mathrm{~F}$ & $4 \mathrm{~d}$ & 7 dias & Meningococemia & Meningococemia & V \\
\hline
\end{tabular}

$\mathrm{a}=$ anos; $\mathrm{AVCI}=$ acidente vascular cerebral isquêmico; síndrome CINCA = síndrome articular infantil, crônica, cutânea e neurológica; $\mathrm{d}=$ dias; $\mathrm{DMJ}=$ dermatomiosite juvenil; $\mathrm{F}=$ feminino; $\mathrm{HCL}=$ histiocitose das células de Langerhans; $\mathrm{HP}=$ hipertensão pulmonar; $\mathrm{IA}=$ idade atual; $\mathrm{IH}=$ intra-hospitalar; $\mathrm{m}=$ meses; $\mathrm{M}=$ masculino; $\mathrm{N}=$ número do paciente; $\mathrm{NA}=$ não aplicável; $\mathrm{PAA}=$ placa de ateroma na aorta; $\mathrm{RP}=$ retroperitoneal; $\mathrm{S}=$ sexo; $\mathrm{SAF}=$ síndrome do anticorpo antifosfolípide; $\mathrm{SNC}=$ sistema nervoso central; $\mathrm{TC}=$ tireoidite crônica; TD = tempo de doença; TI = tempo de internação

Discordância entre o diagnóstico clínico e a necropsia (Classe I, II, III ou IV de Goldman) foi evidenciada em seis casos com LESJ. As infecções fúngicas identificadas, exclusivamente, na necropsia foram: aspergilose difusa no caso
15, aspergilose pulmonar no caso 10 e miocardite fúngica por candida albicans no caso 7 (Tabela 2). Uma paciente, sem epidemiologia para tuberculose, apresentou inicialmente perfuração intestinal com vasculite, evoluindo com 
pleurite, vasculite cutânea, pneumonia, distúrbio do comportamento e coma. O exame do líquor proveniente da punção lombar foi normal. A necropsia evidenciou tuberculose miliar com granuloma e reação Ziehl-Neelsen positiva em: meninges, pulmões, medula óssea, peritônio, fígado, baço, trato gastrintestinal, endométrio, ovários e tubas uterinas (Classe I). Nesta paciente (caso 12) e na paciente de número 9 (Tabela 2) foi evidenciada placa ateromatosa em aorta, sendo que na primeira ocorreu obstrução em mais de $50 \%$ do lúmen da aorta abdominal. No caso 13, que apresentava hematúria isolada e persistente, foi identificada nefrite Classe IV da OMS, somente após necropsia. Esta paciente foi classificada como Classe III de Goldman, sem impacto no seu prognóstico, pois apresentava hemorragia pulmonar e recebeu o tratamento com pulsoterapia com metilprednisolona e ciclofosfamida.

Sete pacientes com AIJ evoluíram para óbito. A causa clínica do óbito foi: septicemia em três, síndrome de ativação macrofágica (SAM) em três e insuficiência cardíaca congestiva por hipertensão pulmonar em um paciente. A idade deles na época do óbito variou de 4 a 18,2 anos (média de 14 anos) e a duração da doença variou de 2 a 12,7 anos (média de 8,7 anos) (Tabela 3).

Três pacientes com AIJ forma sistêmica apresentaram SAM com manifestações agudas de febre alta prolongada, hepatoesplenomegalia, sangramentos, adenomegalia generalizada e icterícia. Estes evoluíram com insuficiência hepática aguda, coma, coagulação intravascular disseminada e falência de múltiplos órgãos. Anemia, leucopenia, plaquetopenia, hipofibrinogenemia, hipertrigliceridemia e alargamentos dos tempos de coagulação ocorreram nos três casos. Os resultados da necropsia mostraram, no caso 2, diátese hemorrágica, esplenite, fibrose periportal e esteatose hepática; no caso 3, diátese hemorrágica, pericardite crônica, esplenite, insuficiência renal aguda e necrose hepática. Nestes dois casos não foi evidenciada hemofagocitose. No caso 1 foram identificadas: diátese hemorrágica, necrose maciça hepática (compatível com hepatite fulminante) e proliferação macrofágica nos pulmões com hemofagocitose. Todos os casos com SAM apresentaram velocidade de hemossedimentação (VHS) abaixo de $15 \mathrm{~mm}$ na primeira hora.

Uma paciente com diagnóstico de AIJ forma sistêmica (artrite crônica, febre característica, exantema reumatóide e pericardite) apresentou curso poliarticular ativo há oito anos e usou metotrexato e ciclosporina (Caso 7, Tabela 3). Esta evoluiu com esclerodermia cutânea linear, hipertensão pulmonar grave (pressão sistólica de artéria pulmonar de $120 \mathrm{mmHg}$ ), insuficiência cardíaca congestiva e morte súbita na UTI. A necropsia evidenciou infarto antigo discreto, dilatação das câmaras cardíacas direitas, derrame pericárdico e aterosclerose generalizada. Surpreendentemente, na necropsia foi também identificado um linfoma não Hodgkin difuso de pequenas células, acometendo alguns linfonodos abdominais e com imunoexpressão positiva para CD 20.

O Staphylococus aureus foi isolado em culturas de três pacientes com AIJ e septicemia; Klebsiella pneumoniae foi isolada simultaneamente em um destes casos. Outros agentes encontrados foram: Candida albicans em um e Corynebacterium sp em outro paciente.

Os demais óbitos ocorreram por outras doenças, as quais estão descritas na Tabela 4.

\section{DISCUSSÃO}

Por ser um serviço terciário, o Instituto da Criança atende crianças e adolescentes gravemente enfermos procedentes de São Paulo, de outras cidades ou Estados do país, dispondo para isto de diversas enfermarias hospitalares.

A melhora no prognóstico das doenças reumatológicas pediátricas nas últimas duas décadas reflete o seu melhor reconhecimento, com diagnóstico e tratamento precoces ${ }^{(17)}$. Por sua vez, estas doenças são graves, de evolução crônica, com intercorrências clínicas e necessidade de múltiplos tratamentos imunossupressores, aumentando o risco de óbito.

Em estudo que avaliou a freqüência das doenças reumatológicas pediátricas em um ambulatório de um hospital terciário $^{(18)}$, observou-se que os diagnósticos mais freqüentes foram: AIJ em 53\% dos casos, espondiloartropatias em 13\%, vasculites em 10\% e LESJ em 6\%. Não há estudos de prevalência em casos internados com doenças reumatológicas pediátricas. Os óbitos na faixa etária pediátrica são descritos como relato de caso. $\mathrm{Na}$ década avaliada neste estudo, a taxa de óbitos nos pacientes internados foi de 10\%, tendo ocorrido o óbito em $85 \%$ dos casos na UTI desse Hospital Universitário.

Os estudos de freqüência de óbitos nas doenças reumatológicas são, habitualmente, descritos na população de adultos. Nos anos 1999 e 2000, Erdes et al. ${ }^{(19)}$ avaliaram 38 serviços de patologia na cidade de Moscou e evidenciaram que $1,8 \%$ das necropsias (784 casos) ocorreram em doenças reumáticas. As mais relacionadas ao óbito foram: artrite reumatóide em 49\%, espondiloartropatias em 10\%, vasculites em 9\%, LES em 12,7\% e osteoartrose em 1,3\%.

Neste estudo, o LESJ foi a principal causa de internação e óbito. A mortalidade de pacientes com LESJ está rela- 
cionada à atividade da doença não controlada por terapia com corticoesteróides e ou imunossupressores. Singh et al. ${ }^{(3)}$ estudaram 31 pacientes indianos com diagnóstico de LESJ por um período de três anos. Destes, 10 evoluíram para óbito (32\%): oito por atividade da doença não controlada, sendo associada à infecção em quatro. Destes, apenas três foram submetidos à necropsia, concordantes com o diagnóstico clínico. Neste estudo, dos 18 casos com LESJ que evoluíram para óbito, 10 (59\%) realizaram necropsia, sendo septicemia associada ou não à atividade da doença a causa determinante do óbito em 16 casos. Estes ocorreram, em média, nos primeiros 26 meses de duração da doença.

Portadores de LESJ são mais susceptíveis as infecções ${ }^{(20,21)}$. As infecções fatais são mais freqüentemente causadas por bactérias $^{(1,2)}$. Neste estudo, as infecções bacterianas ocorreram em 12 casos (70\%), particularmente por Gram positivos (como Staphilococcus aureus de aquisição intra-hospitalar), assim como na literatura ${ }^{(22)}$. Em um estudo nacional, Len et al. ${ }^{(23)}$ diagnosticaram 54 processos infecciosos ocorridos em 34 crianças com LESJ, sendo esta a causa principal de óbito em seis pacientes (18\%): cinco com septicemia e um com tuberculose. Todos estes casos apresentavam atividade da doença. A associação de infecções com nefrite ativa é uma importante causa de óbitos em LESJ ${ }^{(24,25)}$. Sete de nossos pacientes com nefrite prévia tiveram insuficiência renal aguda no momento do óbito, possivelmente multifatorial: atividade da doença, septicemia, choque séptico e falência de múltiplos órgãos.

Em nossa casuística, AIJ foi a segunda doença causadora de morte. Óbito foi evidenciado em sete casos (21\%) com forma sistêmica, tendo sido três por SAM. A mortalidade geral por AIJ, na literatura, varia de $2 \%$ a $4 \%$ sendo mais elevada na forma sistêmica da doença ${ }^{(26,27)}$. As taxas de óbitos variam de 1\% nos Estados Unidos a 4,2\% na Europa, principalmente por amiloidose ${ }^{(28)}$. Bernstein ${ }^{(28)}$ relata que, em pacientes que evoluem para o óbito, insuficiência hepática está presente em 20\% desses. Boone ${ }^{(29)}$ descreve taxas de mortalidade de $17 \%$ do total de 1.674 pacientes com AIJ, por insuficiência hepática, geralmente de causas desconhecidas, sendo excepcionalmente por hepatites virais e uso de sais de ouro, acreditando-se que estes óbitos sejam por SAM. A associação de óbitos por SAM em pacientes com a AIJ sistêmica é de $11 \%$ a 30\%(30,31).

A SAM é uma complicação aguda e grave da AIJ, particularmente de início sistêmico. As alterações laboratoriais incluem pancitopenia, alargamento dos tempos de coagulação, hipofibrinogenemia, elevação do D-dímero, aumento das enzimas hepáticas, redução da VHS, hipoalbu- minemia, hiponatremia e hipertrigliceridemia (>160 mg/ dl), conforme evidenciados nos nossos três $\operatorname{casos}^{(30,31,32,33)}$. Recentemente, a hiperferritinemia (>10.000 mg/dl) tem sido associada com uma positividade na SAM em até 100\% dos pacientes ${ }^{(31)}$. O nosso último caso foi diagnosticado em 1998, época em que a realização desse exame não era de rotina para este diagnóstico. A presença de macrófagos ativos fagocitando hemácias, neutrófilos ou plaquetas no mielograma é evidenciada em até $70 \%$ dos $\operatorname{casos}^{(30,31,32,33)}$. A ausência de proliferação macrofágica na necropsia de dois de nossos casos é decorrente, entre outros fatores, da imunossupressão resultante da própria doença agravada pelo tratamento da SAM.

A iminência do óbito costuma envolver toda a família, requerendo afetividade e proximidade na relação entre familiares e equipe médica. Em nossa casuística obtivemos consentimento de 21 necropsias (64\%). Esta freqüência considerável reflete, provavelmente, o bom relacionamento entre profissionais e familiares que permitiram a realização da necropsia em um momento de sofrimento. A opção pelo tipo de necropsia dependeu da solicitação do corpo clínico e da localidade do óbito. Nos óbitos mal definidos autorizados pelos familiares no Hospital das Clínicas são realizados, habitualmente, necropsias do tipo A e B, como ocorreu em todos os casos do presente estudo.

As necropsias são importantes para determinar eventos não esclarecidos ou duvidosos no óbito. Compreendendose melhor a doença e as circunstâncias da morte, pode-se algumas vezes evitar acontecimentos determinantes para esta e aplicar conhecimentos que resguardariam a vida. Observa-se que, na maioria das vezes, a necropsia fornece pelo menos uma informação que era desconhecida em vida ou algum diagnóstico adicional. Estudos de necropsias em adultos mostram grande acurácia das técnicas forenses em diagnosticar causa de óbito em morte não acidental (causas naturais) ${ }^{(34)}$. As doenças reumatológicas, por serem caracterizadas por inflamação sistêmica e crônica, oferecem dificuldades para diagnósticos precisos quando baseados somente em dados clínicos ${ }^{(35,36)}$.

Estudos de necropsias são mais comumente descritos na faixa etária neonatal( ${ }^{(37)}$, na avaliação de morte súbita nos lactentes $^{(38)}$ e nas UTIs pediátricas ${ }^{(39)}$. No período compreendido entre 1996 e 1998, Cardoso ${ }^{(39)}$ estudou 102 necropsias de pacientes internados na UTI do Instituto da Criança. As principais causas clínicas de óbitos foram: choque séptico em 48\%, insuficiência respiratória em 29\% e septicemia em 11\%. A necropsia revelou achados inesperados em 73 pacientes do estudo (72\%). Em 12 desses pacientes, se 
descobertos em vida, os achados alterariam o seu tratamento e provavelmente o seu prognóstico (Classe I de Goldman).

Discordância entre o diagnóstico clínico e a necropsia foi evidenciada em seis casos com LESJ. As três infecções fúngicas e a tuberculose miliar foram identificadas exclusivamente na necropsia. Placas de ateroma na aorta, de maneira surpreendente, foram evidenciadas na necropsia de dois casos com LESJ, um com AIJ e um com síndrome CINCA. Um outro caso com AIJ apresentava uma aterosclerose difusa com infarto do miocárdio antigo. $\mathrm{Na}$ literatura médica, até o presente momento, não há descrição destes achados em necropsias destas doenças nas crianças e adolescentes. Urowitz et al. ${ }^{(4)}$ mostraram que $13 \%$ de adultos com LES apresentaram sintomas clínicos atribuídos à aterosclerose (angina, infarto do miocárdio e/ou doença vascular periférica). Estudos de necropsia em adultos evidenciaram que até metade dos pacientes com LES têm aterosclerose importante, podendo este ser um achado ocasional ou a causa primária de óbito ${ }^{(2)}$. Como fatores de risco para aterosclerose em nossos cinco pacientes ressaltamos em todos: atividade prolongada da doença, sedentarismo, hipertensão arterial, e uso de corticosteróides (por período prolongado e com altas doses cumulativas). Dois destes casos apresentaram também dislipidemia prévia, com elevação do colesterol LDL ${ }^{(40,41)}$

O paciente portador de LESJ, por ser imunossuprimido, quer pela doença quer por seu tratamento, está também mais propenso às infecções oportunísticas, principalmente por fungos e tuberculose $\mathrm{e}^{(22,42,43,44)}$. Iriya et al. ${ }^{(36)}$ estudaram 200 óbitos em adultos com LES, acompanhados no Hospital das Clínicas da FMUSP, e evidenciaram que 113 (56\%) destes foram submetidos à necropsia. A principal causa de óbito foi infecção, ressaltando a presença de uma ou mais infecções oportunísticas em 15 casos: tuberculose em sete, aspergillus em cinco, criptococcus em três e candida em três. Apesar do procedimento de rotina de nosso serviço em realizar culturas em pacientes internados para bactérias e fungos, assim como pesquisar bacilo álcool ácido resistente e cultura para tuberculose em todos os casos, estes agentes foram identificados apenas nas necropsias, a despeito de

\section{REFERÊNCIAS}

1. Ward MM, Pyun E, Studenski S: Causes of death in systemic lupus erythematosus: Long-term follow-up of an inception cohort. Artritis Rheum 38: 1492-9, 1995.

2. Abu-Shakra M, Urowitz MB, Gladman DD, Gough J: Mortality studies in systemic lupus erythematosus. Results from a single center. I. Causes of death. J Rheumatol 22: 1259-64, 1995.

3. Singh S, Devidayal, Kumar L, Joshi K: Mortality patterns in childhood lupus- 10 years experience in a developing country. Clin Rheumatol 21: 462-5, 2002. dois casos terem recebido terapia anti-fúngica. O uso de pulsoterapia com ciclofosfamida e corticosteróides, catéteres endovenosos, ventilação mecânica e necessidade de múltiplos antibióticos devem alertar para a pesquisa de fungos, indicando tratamento com antifúngicos.

Em uma paciente com AIJ foi evidenciado na necropsia, surpreendentemente, um linfoma não Hodgkin em fase inicial. Esta paciente recebeu imunossupressores para o controle de sua doença (metotrexato e pulsoterapia com ciclofosfamida). Raros casos de associação de AIJ e linfoma não Hodgkin com uso de metotrexato têm sido descritos ${ }^{(45)}$.

Dentre as doenças raras que evoluíram para óbito em nossos casos ressaltamos a síndrome CINCA e a síndrome de Churg-Strauss. As principais causas de óbito relacionadas à síndrome CINCA são: leucoencefalopatia necrosante ${ }^{(46)}$, septicemia ${ }^{(47)}$ e amiloidose ${ }^{(48)}$. Esta última ocorreu em nosso caso e foi diagnosticada apenas na necropsia. A síndrome de Churg-Strauss é uma vasculite rara na faixa etária pediátrica, com cerca de oito casos publicados em crianças e adolescentes. $\mathrm{Na}$ faixa etária pediátrica, os óbitos estiveram relacionados, principalmente, com comprometimento cardíaco e gastrintestinal ${ }^{(49)}$. O paciente deste estudo apresentou movimentos involuntários coréicos por vasculite de SNC com evolução para coma e falência de múltiplos órgãos.

A necropsia é importante para determinar eventos não esclarecidos ou duvidosos no óbito e deve ser sempre solicitada. Neste estudo, doenças infecciosas, aterosclerose e/ ou neoplasia foram evidenciadas, exclusivamente, na necropsia e não foram diagnosticadas previamente. Estes achados poderiam modificar a conduta terapêutica e, possivelmente, o prognóstico destas doenças.

\section{AGRADECIMENTOS}

Os autores agradecem às Dras. Maria Helena Bitencourt Kiss, Bernadete de Lourdes Liphaus, Lucia Maria Mattei Arruda Campos; Cristina Miuki Abe Jacob, Vera Herminia Koch e às equipes do Pronto Socorro e UTI do ICr-HC-FMUSP o acompanhamento conjunto de alguns casos desta casuística.

4. Urowitz MB, Gladman DD: How to improve morbidity and mortality in systemic lupus erythematosus. Rheumatology 39: 238-44, 2000.

5. Hochberg MC: Updating the American College of Rheumathology revised criteria for the classification of systemic lupus erythematosus. Arthritis Rheum 40: 1725, 1997.

6. Masi AJ, Hunder GG, Lie JT, et al: The American College of Rheumatology 1990 criteria for the classification of Churg-Strauss syndrome. Arthritis Rheum 33: 1094, 1990.

7. Bohan A, Peter JB: Polymyositis and dermatomyositis. N Engl J Med 292: 344-403, 1975. 
8. Petty RE, Southwood TR, Manners P, et al: International League of Associations for Rheumatology classification of juvenile idiopathic arthritis: second revision, Edmonton, 2001. J Rheumatol 31: 390-2, 2004.

9. Weening JJ, D'Agati VD, Schwartz MM, et al: The classification of glomerulonephritis in systemic lupus erythematosus revisited. Kidney Int. 65: 521-30, 2004.

10. Bombardier C, Gladman DD, Urowitz MB, Karon D, Chang CH: Derivation of the SLEDAI. A disease activity index for lupus patients. The Committee on Prognosis Studies in SLE. Arthritis Rheum 35: 630-40, 1992.

11. Barr SG, Zonana-Nacach A, Magder LS, Petri M: Patterns of disease activity in systemic lupus erythematosus. Arthritis Rheum 42: 2682-8, 1999.

12. Gladman D, Ginzler E, Goldsmith C, et al: The development and initial validation of the Systemic Lupus International Collaborating Clinics/American College of Rheumatology Damage Index for systemic lupus erythematosus. Arthritis Rheum 39: 363-9, 1996.

13. Hanson V, Kornreich H, Bernstein B, King KK, Singsen BH: Prognosis of juvenile rheumatoid arthritis. Arthritis Rheum (Supllement) 20: 279-84, 1977.

14. Ramos SR. Epidemiologia das Infecções Hospitalares em Pediatria. In: Marcondes E, Vaz AC, Ramos JL, Okay Y: Pediatria Básica Tomo II, 9.a ed, São Paulo, Sarvier, 2003, p. 92-5.

15. Macperson TA, Stocker JT. The pediatric autopsy. In: Stocker JT, Dehner LP. Pediatric Pathology, Philadelphia, Lippincott Williams e Wilkins,1992, p. 3-14

16. Goldman L, Sayson R, Robbins S, Cohn LH, Bettmann M, Weisberg RN: The value of the autopsy in three medical eras. N Engl J Med 308: 1000-5, 1983

17. Jacobsen S, Petersen J, Ullman S, et al: Mortality and causes of death of 513 danish patients with systemic lupus erythematosus. Scand J Rheumatol 28: 75-80, 1990.

18. Bethany AD, Lori BT, Laurie CM, et al: Demography of a Regional Pediatric Rheumatology Patient Population. J Rheumatol 21: 1553-7, 1994.

19. Erdes SH, Demina AB, Folomeeva OM, Radenska-Lopovok SG, Zairat"iants OV: Analysis of lethal outcomes of rheumatic disease in Moscow. Ter Arkh 75: 78-82, 2003.

20. Lacks S, White S: Morbidity associated whit childhood systemic lupus erythematosus. J Rheumatol 17: 941-5, 1990.

21. Pizzo PA: Fever in the immunocompromissed patients. N Engl J Med 341: 893-900, 1999.

22. Petri M: Infection in systemic lupus erythematosus. Rheum Dis Clin North Am 24: 423-56, 1998.

23. Len CA, Terreri MT, Hilário MOE: Lúpus eritematoso sistêmico juvenil e infecção. Rev Bras Reumatol 42: 218-22, 2002.

24. Cassidy JT, Sullivan DB, Petty RL, Rogsdale C: Lupus nephritis and encephalopathy. Prognosis in 58 children. Arthritis Rheum 20: 315-22, 1977.

25. Lee BW, Yap HK, Yip YC: A 10 years review of systemic lupus erythematosus in Singapore children. Aust Pediatr J 23: 163-5, 1987.

26. Baum J, Gutowska G: Death in juvenile rheumatoid arthritis. Arthritis Rheum (Supplement) 20: 253-5, 1977.

27. Minaen K, Nierwerth M, Listing J, et al: Long-term outcome in patients with juvenile idiopatic arthritis. Arthritis Rheum 46: 2392-401, 2002.

28. Bernstein B: Death in juvenile rheumatoid arthritis. Arthritis Rheum (Supplement) 20: 256, 1977.
29. Boone JE: Hepatic disease and mortality in juvenile rheumatoid arthritis. Arthritis Rheum (Supplement) 20: 257-9, 1977.

30. Grom AA, Passo M: Macrophage activation syndrome in systemic juvenile rheumatoid arthritis. J Pediat 129: 630-2, 1996.

31. Ravelli A: Macrophage activation syndrome. Curr Opin Rheumatol 14: 548-52, 2002.

32. Stéphan JL, Koné-Paut I, Galambrun C, Mouy R, Bader-Meunier B, Prieur AM: Reactive haemophagocytic syndrome in children with inflammatory disorders. A retrospective study of 24 patients. Rheumatol 40: 1285-92, 2001.

33. Stephan JL, Zeller J, Hulert P, et al: Macrophage activation syndrome and rheumatic disease in childhood: A report of four new cases. Clin Exp Rheumatol 11: 451-6, 1993.

34. Aran BM, Perez G, Rosell J, Molina P: Exactness of mortality statistics by external and natural cause of death with medico-legal intervention in Catalônia. Gac Sanit 14 : 356-62, 2000.

35. Suzuki A, Ohosone Y, Obana M, et al: Cause of death in 81 autopsied patients with rheumatoid arthritis. J Rheumatol 21: 33-6, 1994.

36. Iriya SM, Capelozzi VL, Calich I, Martins MA, Lichtenstein A: Causes of death in Patients With Systemic Lupus Erythematosus in São Paulo, Brazil: A Study of 113 Autopsies. Arch Intern Med 161: 1557-60, 2001.

37. Hunt R, Barr P: Errors in the certification of neonatal death. J Paediatr Child Health 36: 498-501, 2000.

38. Hatton F, Bouvier-Colle MH, Barois A, et al: Autopsies of sudden infant death syndrome-classification and epidemiology. Acta Paediatr 84: 1366-71, 1995.

39. Cardoso MP: Concordância entre diagnóstico clínico e achados de autópsias em unidade de terapia intensiva pediátrica. Dissertação de Mestrado. Faculdade de Medicina da Universidade de São Paulo, 2000.

40. Manzi S, Meilahn EN, Raine JE, et al: Age specific incidence rates of myocardial infarction and angina in women with systemic lupus erythematosus: comparison with the Frammingham cohort. Am J Epidemol 145: 408-415, 1997.

41. Dória A, Shoenfeld Y, Wu R, et al: Risk factors for subclinical atherosclerosis in a prospective cohort of patients with systemic lupus erythematosus. Ann Rheum Dis 62: 1071-7, 2003.

42. Canova EG, Rosa DC, Vallada MG, Silva CA: Invasive aspergillosis in juvenile systemic lupus erythematosus. A clinico-pathologic case. Clin Exp Rheumatol 20: 736, 2002.

43. Pryor BD, Bologna SG, Kahl LE: Risk factors for serious infection during treatment with cyclophosphamide and high dose of corticosteroids for systemic lupus erythematosus. Arthritis Rheum 39: 1475-82, 1996.

44. Ruben LA, Urowitz MB, Gladman DD: Mortality in systemic lupus erythematosus: The modal pattern revisited. QJ Med 55: 87-98, 1985.

45. Cleary AG, McDowell H, Sills JA: Polyarticular juvenile idiopathic arthritis treated with methotrexate complicated by the development of non-Hodgkin's lymphoma. Arch Dis Child 86: 47-9, 2002.

46. Lampert F, Belohradsky BH, Forster C: Infantile chronic relapsing inflammation of the brain, skin and joints. Lancet 1: 1250, 1975.

47. Fajardo JE, Geller TJ, Koenig HN: Chronic meningitis, polyarthritis, lymphadenitis and pulmonary hemosiderosis. J Pediatr 101: 738, 1982.

48. Prieur AM, Griscelli C, Lampert F: A chronic, infantile, neurological cutaneous and articular (CINCA) syndrome. A specific entity analyzed in 30 patients. Scand J Rheumatol 66: 57, 1987.

49. Cassidy JT, Petty RE: Juvenile rheumatoid arthritis. In: Cassidy JT, Petty RE: Textbook of Pediatric Rheumatology, 4 ed, Philadelphia, WB Saunders Company, 2001, p. 218-321. 